\title{
Comprehensive Medication Reviews in Long-term Care Facilities: History of Process Implementation and 2015 Results
}

\author{
Terrence E. O'Shea, PharmD, CGP; Barbara J. Zarowitz, PharmD, CGP, BCPS; \\ and W. Gary Erwin, PharmD
}

\begin{abstract}
BACKGROUND: Since 2013, Part D sponsors have been required to offer comprehensive medication reviews (CMRs) to all beneficiaries enrolled in their medication therapy management (MTM) programs at least annually, including those in long-term care (LTC) settings. Since that time, MTM providers have found that accessing and completing CMRs with beneficiaries is frequently prohibitively complex, since the process often requires a live, face-to-face interactive interview where the beneficiary resides. However, with the migration of the CMR completion rate from a star ratings display measure to an active measure, coupled with the new CMR completion rate cutpoints for 2016, accessing this population for CMR completion has heightened importance.

PROGRAM DESCRIPTION: Our proprietary consultant pharmacist (CP) software was programmed in $\mathbf{2 0 1 2}$ to produce a cover letter, medication action plan, and personal medication list per CMS standardized format specifications. Using this system, CPs were trained to perform and document CMRs and the interactive interviews. MTM-eligible Part $D$ beneficiaries, identified by several contracted clients as residing in LTC serviced by 0mnicare, were provided CMRs and summaries written in CMS standardized format by CPs. Residents with cognitive impairment were identified using 3 data elements in the Minimum Data Set (MDS).
\end{abstract}

OBSERVATIONS: In 2015, 7,935 MTM-eligible beneficiaries were identified as receiving medications from an Omnicare pharmacy. After excluding those who were disenrolled by their prescription drug plans, discharged from the LTC facility, or resided in a LTC facility no longer serviced by Omnicare, 5,593 residents were available for CMR completion. Of these, only $3 \%$ refused the CMR offer, and 5,392 CMRs (96\%) were completed successfully. Thirty-nine percent of residents had cognitive impairment per MDS assessments; in those instances, CMRs were conducted with someone other than the beneficiary. Based on the CMRs and interactive interviews, 7,527 drug therapy problem recommendations were made to prescribers, about $50 \%$ of which resulted in an alteration in therapy, including reductions in polypharmacy and high-risk medications.

IMPLICATIONS: The CMR process and written summary in CMS standardized format works effectively for residents in LTC when performed by CPs in the facility, as evidenced by high completion rates and drug therapy problem identification/resolution. Part D plans should further consider using CPs to conduct CMRs in LTC settings.

J Manag Care Spec Pharm. 2017;23(1):22-26

Copyright $\odot 2017$, Academy of Managed Care Pharmacy. All rights reserved.

\section{What is already known about this subject}

Comprehensive medication reviews (CMRs) have been conducted in a variety of health care settings, but they have only been required for nursing home residents since 2013.

CMR completion rates are low nationally (20\% in 2015).

Nursing homes are self-contained communities within which familiar personnel are welcome.

\section{What this study adds}

CMR completion rate can be high (96\%) when conducted using a systematic approach and delivered by consultant pharmacists in their own facilities.

The majority of nursing home residents (63\%) were able to participate in CMR interviews.

Refusal by long-term care residents to participate in CMR interviews rarely occurred (3\%), suggesting the need for an impetus to increase the CMR focus in this population, which is at high risk for medication problems.

$\mathrm{M}$ edication therapy management (MTM) is a patientcentered, comprehensive approach to improve medication use, reduce the risk of adverse events, and improve medication adherence. ${ }^{1}$ Targeted beneficiaries for MTM programs encompass patients who have multiple chronic diseases (e.g., heart failure, diabetes, and respiratory disease), take multiple Part D-covered medications, and are likely to incur costs for covered Part D medications that are greater than or equal to the 2015 costs of $\$ 3,138 .{ }^{1}$ Part D plan sponsors are required to offer (a) interventions for beneficiaries and prescribers, (b) annual comprehensive medication reviews (CMRs) with written summaries according to CMS standardized format specifications, and (c) quarterly targeted medication reviews with follow-up interventions as necessary.

In April 2012, the Centers for Medicare \& Medicaid Services (CMS) mandated that, beginning in 2013, plan sponsors must offer CMRs to all beneficiaries enrolled in the MTM program at least annually, including beneficiaries in long-term care (LTC) settings, who were previously exempted from these requirements. CMS defines a CMR as "a systematic process of collecting patient-specific information, assessing medication therapies to identify medication-related problems, 
developing a prioritized list of medication-related problems, and creating a plan to resolve them with the patient, caregiver, and/or prescriber."' Additionally, CMS specifies that each CMR must include an interactive, person-to-person, or telehealth medication review and consultation of all of the beneficiary's medications (i.e., prescription and over-the-counter medications, herbal therapies, and dietary supplements). This interaction must occur in real time with a pharmacist or other qualified MTM provider, and a written summary of the results of the review must be provided to the beneficiary. The CMS standardized format that is provided to the beneficiary consists of (a) a cover letter that describes the CMR encounter; (b) a medication action plan (MAP), which outlines key medicationrelated issues identified in the interactive interview and specific actions the patient should take related to those issued; and (c) a personal medication list (PML), which is a list of the patient's current medications. ${ }^{2}$

Realizing that some LTC beneficiaries are cognitively impaired and not able to take part in the interactive interview, CMS recommends conducting the CMR interview with the beneficiary's prescriber, caregiver, or other authorized individual (e.g., legal guardian or health care proxy). It is incumbent upon the Part D plan sponsor to provide documentation of the determination of cognitive impairment for each applicable beneficiary.

Pursuant to the new requirement to provide CMRs in the LTC setting, Omnicare began contracting with Part D plans and pharmacy benefit management companies in 2013 to provide CMRs to targeted beneficiaries residing in skilled nursing facilities. The history of process development, implementation, and results of these efforts by Omnicare consultant pharmacists (CP) during the calendar year (CY) 2015, are described in this article.

\section{Program Description}

Subsequent to the release of the CMS CY 2013 MTM program guidance and submission instructions, we conducted a thorough analysis of the new requirements, with particular emphasis on the CMS standardized format. Through a collaborative effort between individuals from the business, clinical, and information technology groups within Omnicare, the standardized cover letter, MAP, and PML were programmed into our proprietary CP decision support software. In addition, based on our extensive experience and knowledge of the LTC arena and the Minimum Data Set (MDS) resident assessment instrument used in nursing homes, we developed a working definition of how to identify cognitively impaired residents. This definition was based on 3 elements of the MDS (version 3.0): (1) item C0500-Brief Interview for Mental Status (BIMS) summary score (a validated instrument for assessing cognition in nursing home residents ${ }^{3}$ ), (2) item B0700 ("Makes Self Understood"), and (3) item 0800 ("Ability To Understand
Others"). If the BIMS summary score was 0-7 (indicative of severe cognitive impairment); or item B0700 was scored as a 2 or 3 (2 ="sometimes understood," $3=$ "rarely/never understood"); or item B0800 was scored as a 2 or 3 ( $2=$ "sometimes understands," 3 = "rarely/never understands"), then the resident was considered to be cognitively impaired and thus not a candidate to participate in the interactive interview. Subsequent to our development of these criteria in late 2012, CMS adopted the inclusion of this approach to determining cognitive impairment in their Medication Therapy Management Program Guidance and Submission Instructions memos beginning in 2014. ${ }^{4-6}$ After sufficient testing of the support software and training of the $\mathrm{CP}$, a pilot program was conducted for approximately 200 nursing home residents in December 2012.

After successful completion of the pilot, an agreement was reached with a large national health plan for the Omnicare CP to provide MTM services (CMRs) for their beneficiaries in 2013. Once these beneficiaries were identified by the plan as eligible for MTM services, a matching algorithm was used to identify these residents in our system to determine if the resident was still active in one of our serviced facilities. After CPs were trained in MTM services, the new CMS requirements, and how to document MTM services in our decision support software, MTM services were initiated in March 2013. Since that initial implementation, we have contracted with numerous additional health plans, as well as a pharmacy benefits management company, and continue to add new clients.

\section{Observations}

In 2015, 9,670 MTM-eligible beneficiaries were identified by our contracted clients as receiving service from an Omnicare pharmacy. Of these, 7,935 beneficiaries were identified as currently or recently residing in Omnicare-serviced nursing facilities, 5,593 of whom were actually in the facilities when CPs attempted to conduct CMRs. Of the 5,593 allocated residents still residing in an Omnicare-serviced facility, 5,392 (96\%) participated in CMRs (see Figure 1). One hundred percent of the 5,392 CMRs were conducted by CPs nationally at for-profit and not-for-profit facilities. Although there was a wide geographic distribution of completed CMRs, the highest concentrations were in the Northeast, Southeast, and Midwest. The mean age of residents was 79 years; the median age was 81 years; and $68 \%$ were female. Twelve percent of the CMR beneficiaries were aged less than 65 years, while 38\% were aged 85 years or older.

The majority $(n=3,341,62 \%)$ of beneficiaries were identified as cognitively intact. Of these, 642 (19\%) requested that the interactive interview be conducted with someone else, but half of the CMRs were able to be completed with the beneficiary. Thirty-eight percent $(n=2,051)$ of the beneficiaries was identified as having cognitive impairment so were unable to 


\section{FIGURE 1 Flow Diagram of CMR-Eligible Beneficiaries}

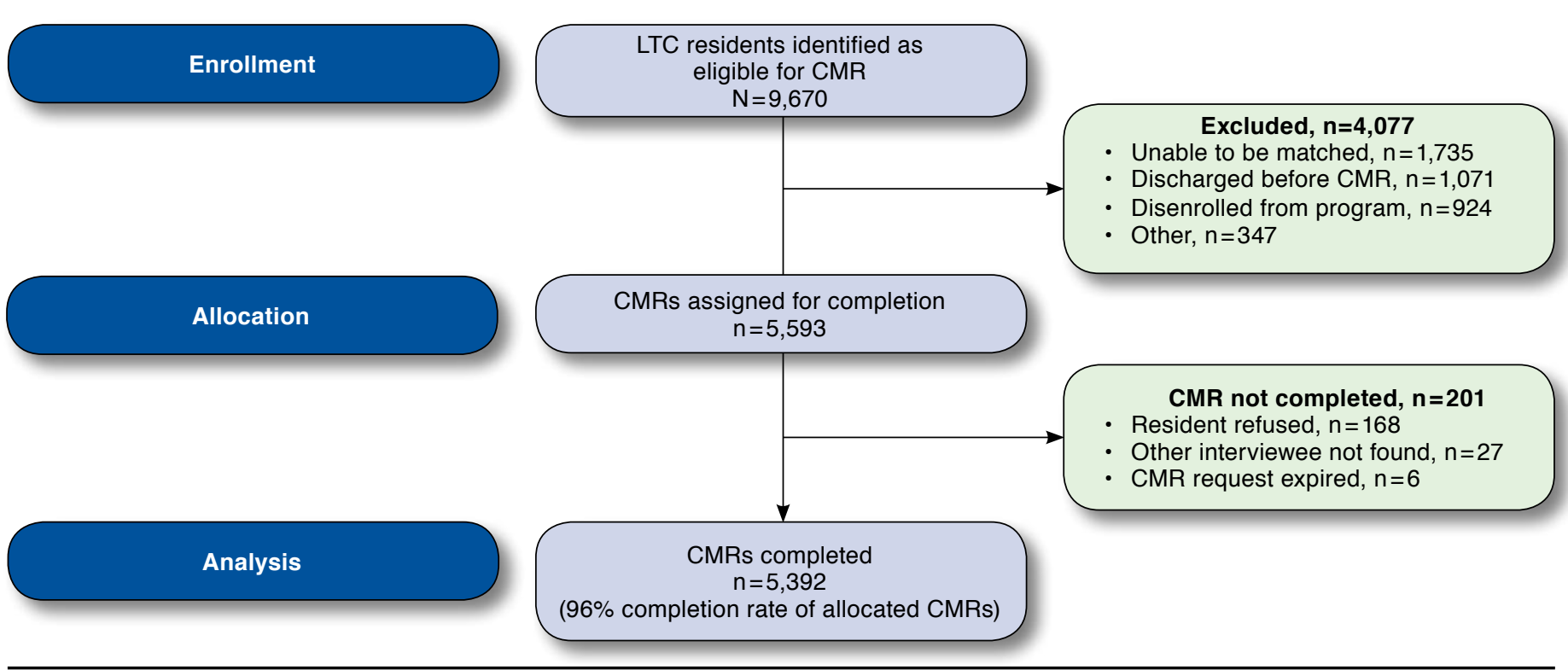

$C M R=$ comprehensive medication review; $L T C=$ long-term care.

participate in the interactive interview. In these instances, the interviews were conducted with the beneficiaries' health care proxies, legal guardians, caregivers, or prescribers (Table 1).

The most common prescriber interventions for drug therapy problems included polypharmacy reduction, dosage adjustment, monitoring, and indication documentation related most often to psychopharmacologic, analgesic, antidiabetic, anticoagulant, and respiratory medication use. Approximately $50 \%$ of pharmacists' recommendations regarding drug-related problems (DRP) resulted in an alteration in therapy. The most common DRP resolutions were "drug discontinued," "dose decreased," and "medication changed."

\section{Implications}

In 2013, the additional CMS requirement that CMRs be conducted for MTM-eligible beneficiaries in LTC settings presented challenges and opportunities. One of the biggest challenges for managed care pharmacy was to locate eligible individuals in LTC facilities; however, the greater challenge then became how to provide CMRs for these individuals (or other cognitively impaired beneficiaries). The dynamics of LTC facilities are often not conducive to the telephonic provision of CMRs because of privacy and security concerns and the general logistics of having a LTC resident participate in a lengthy phone call. Providing CMRs in this setting is most effective when done in a face-to-face format, which is also problematic for many managed care/MTM providers, who are often providing these services from a centralized call center. The ideal individual to provide these services in this setting is the LTC facility's CP, who is required by CMS to perform medication regimen reviews (MRR) on at least a monthly basis so is in the facility frequently.

Using the facility's $\mathrm{CP}$ also gives the $\mathrm{CP}$ certain advantages, such as knowing the residents and their histories and current statuses, based on previous MRR activities. Other advantages include having real-time medication information and laboratory results, as well as having a relationship with the resident's prescribers, which may allow for more effective prescriber interventions.

For our company, one of the particularly challenging aspects of implementing our program was developing a workable mechanism that enabled us to meet the rigorous formatting requirements of the CMS standardized format. We were able to accomplish this by programming the 3 documents (Cover letter, MAP, and PML) into our proprietary CP decision-support software. Other challenges that were successfully overcome included CP training, locating other individuals with whom to conduct CMRs when the beneficiary was unable to participate, and the distribution of CMR documents. Among the numerous opportunities afforded by this new requirement was the opportunity to improve resident care and understanding of their medications.

While we are unaware of any specific articles in the managed care literature regarding the implementation of a program of providing CMRs to beneficiaries in LTC settings, Perlroth et al. (2013), in a report to CMS in 2013, ${ }^{7}$ investigated how Part D 


\begin{tabular}{|c|c|c|}
\hline Characteristic & Number & Percentage \\
\hline Female & 3,662 & 68 \\
\hline Male & 1,730 & 32 \\
\hline \multicolumn{3}{|l|}{ Age range (years) } \\
\hline$<65$ & 637 & 12 \\
\hline $65-74$ & 1,042 & 19 \\
\hline $75-84$ & 1,668 & 31 \\
\hline$>85$ & 2,045 & 38 \\
\hline Cognitive impairment & 2,051 & 38 \\
\hline CMR completed with beneficiary & 2,699 & 50 \\
\hline \multicolumn{3}{|c|}{ Interviewee when beneficiary unable to complete interactive interview } \\
\hline Prescriber & 948 & 35 \\
\hline Caregiver & 676 & 25 \\
\hline $\begin{array}{l}\text { Other authorized individual } \\
\text { (e.g., health care proxy, legal guardian) }\end{array}$ & 1,069 & 40 \\
\hline$C M R=$ comprehensive medication review. & & \\
\hline
\end{tabular}

MTM programs in operation in 2010 affected adherence, quality of prescribing, resource utilization, and cost of hospital and emergency room care of community-based Medicare beneficiaries. This report specifically analyzed how enrollment in an MTM program, with or without CMRs, influenced medication adherence, quality of prescribing, resource utilization, and costs for Medicare beneficiaries with congestive heart failure, chronic obstructive pulmonary disease, or diabetes. This research demonstrated that improvements in drug therapy outcomes from MTM enrollment were "robust and persistent for adherence and the use of evidence-based medications." " Perlroth et al. found that MTM enrollees who received CMRs were more likely to experience increases in medication adherence and improvements in quality of prescribing, suggesting that CMRs may be one of the more important components of the MTM program. ${ }^{7}$

In addition, beneficiaries with congestive heart failure and diabetes who were newly enrolled in MTM and participated in CMRs consistently had lower risks of hospitalizations and emergency room visits, resulting in hospital cost savings. It is the hope that similar benefits will be realized by beneficiaries who receive CMRs in the LTC setting, an issue that may be addressed by future research initiatives.

Unfortunately, while opportunities for CPs to become involved with providing CMRs were afforded by the 2013 CMS mandate, a relatively small number of beneficiaries in LTC are currently provided these services, since many MTM vendors employ telephonic, call-center-based MTM platforms that are unfamiliar and extremely cumbersome, at best, in the LTC environment.

More recently, a CMS fact sheet stated that, as of April 2016, there were 623 Medicare Part D contracts with an approved MTM program and that all sponsors required to establish an MTM program had an approved program in place. ${ }^{8}$ Of these contracts, 495 (79\%) were Medicare Advantage prescription drug plans; 67 (11\%) were stand-alone prescription drug plans; and 61 (10\%) were Medicare-Medicaid plans. While only 1 component of a plan's MTM program, the provision of CMRs and the corresponding CMR completion rate for eligible beneficiaries has achieved a heightened importance in 2016, with its addition as an active measure to the Medicare Part D star ratings for Medicare plans. Despite the historically low CMR completion rate by plan sponsors (20\% average plan performance for the 2015 CMR completion rate display measure), ${ }^{9}$ CMS established aggressive cutpoints for achieving high star ratings in this measure (published elsewhere) for CY 2016. ${ }^{10}$ Plans with low CMR completion rates and/or aggressive star ratings targets for this measure may benefit from using CPs to conduct CMR interviews in LTC facilities.

The CMS fact sheet also reported that nearly $25 \%$ of Part D MTM programs used expanded eligibility requirements beyond the CMS minimum requirements. ${ }^{8}$ Diabetes, chronic heart failure, dyslipidemia, and hypertension remained the top targeted diseases in 2016 , but $13.5 \%$ of the MTM programs allow any Part D drug to qualify in order to establish the requirement that beneficiaries take multiple Part D drugs, and $74.5 \%$ require Part D drugs for chronic conditions. Of note is the increase in the percentage of Part D sponsors that offered face-to-face CMRs in 2016 (75\%) compared with those offered in 2015 (57.5\%). All MTM programs use pharmacists to provide services, and an increasing percentage of programs have contracted with outside vendors. Given the multiple characteristics of LTC residents and their living environment that make telephonic approaches impractical or infeasible, face-to-face CMRs may become a standard approach for conducting CMRs in this population of vulnerable older adults.

In 2015, CMS proposed changes for MTM services that would have substantially broadened the MTM-eligible population through revision of (a) the definition of "multiple chronic diseases" to require that plan sponsors must target beneficiaries having 2 or more chronic diseases; (b) the definition of "multiple Part D drugs" to require that sponsors target beneficiaries receiving 2 or more Part D covered drugs; and (c) the annual cost threshold change to $\$ 620 .{ }^{11}$ Based on these proposed criteria, it was estimated that $55 \%$ of Part D beneficiaries would be eligible for MTM services. ${ }^{11}$ These changes would have translated into a significantly increased number of beneficiaries eligible for CMRs and thus increased opportunities for CPs to provide these reimbursable cognitive services to residents in LTC facilities. Unfortunately, these proposed revisions were not implemented; however, CMS has stated that it would revisit MTM changes in future rules.

Unexpected findings from the implementation of our program showed that $63 \%$ of nursing home residents were able to participate in the CMR interview process and that $12 \%$ of 
residents who received CMRs were aged less than 65 years. In addition, only 168 (3\%) refusals to participate in a CMR occurred, which we found to be surprisingly low. Notwithstanding the challenges of providing CMRs to LTC residents, CPs communicated an overall positive experience with the process through increased interpersonal communication with residents and/or their families and other interdisciplinary team members, as well as knowing that reimbursement for these services was being provided. While several publications have cited the provision of MTM and CMR services as potentially significant opportunities for $\mathrm{CPs},{ }^{12,13}$ this report describes the actual implementation of such a program and realization of these opportunities in a real-world LTC setting.

\section{Summary}

Despite the operational and logistical challenges of providing CMRs to MTM-eligible Part D beneficiaries in nursing homes in a very specific format mandated by CMS, a focused, collaborative effort between Omnicare and several national clients has enabled the successful implementation and execution of this program, with a CMR completion rate of $96 \%$ for allocated beneficiaries in CY 2015. CPs indicated that the Omnicare CMR process favorably affected resident care and medication understanding, and the process was well received by residents and other CMR recipients. Provision of CMRs by CPs is an additional means for achieving payment for cognitive services and is an additional means by which important interprofessional team members can help improve resident quality of life and function.

\section{Authors}

TERRENCE E. O'SHEA, PharmD, CGP; BARBARA J. ZAROWITZ, PharmD, CGP, BCPS; and W. GARY ERWIN, PharmD, Omnicare, Cincinnati, Ohio.

AUTHOR CORRESPONDENCE: Terrence E. O'Shea, PharmD, CGP, Omnicare, 600 Union Blvd., Englewood, OH 45322.

Tel.: 937.771.2484; Fax: 937.836.1209; E-mail: terry.oshea@omnicare.com.

\section{DISCLOSURES}

No outside funding supported this research. All authors are employees of Omnicare, a CVS Health Company, and are stockholders of CVS Health. O'Shea and Zarowitz have received research funding (unrelated to the submitted work) from Acadia, AstraZeneca, and Sunovion.

The abstract for this article was presented as a research poster at the Academy of Managed Care and Specialty Pharmacy 2016 Annual Meeting; April 21, 2016; San Francisco, California.

Study concept and design were contributed by O'Shea and Zarowitz, along with Erwin. O'Shea collected the data, and data interpretation was performed primarily by O'Shea, along with Zarowitz and Erwin. The manuscript was written by O'Shea, along with Zarowitz, and revised primarily by Zarowitz, along with O'Shea and Erwin.

\section{REFERENCES}

1. Tudor CG. CMS Memorandum. CY 2013 medication therapy management program guidance and submission instructions. April 10, 2012. Available at: https://www.cms.gov/medicare/prescription-drug-coverage/prescriptiondrugcovgenin/downloads/memo-contract-year-2013-medication-therapymanagement-mtm-program-submission-v041012.pdf. Accessed

November 29, 2016.

2. Centers for Medicare \& Medicaid Services. MTM program standardized format revisions (v07.15.14). Available at: http://www.cms.gov/Medicare/ Prescription-Drug-Coverage/PrescriptionDrugCovContra/MTM.html. Accessed November 29, 2016.

3. Chodosh J, Edelen MO, Buchanan JL, et al. Nursing home assessment of cognitive impairment: development and testing of a brief instrument of mental status. J Am Geriatr Soc. 2008;56(11):2069-75.

4. Tudor CG. CMS Memorandum. CY 2014 medication therapy management program guidance and submission instructions. April 5, 2013. Available at: https://www.cms.gov/medicare/prescription-drug-coverage/prescriptiondrugcovcontra/downloads/memo-contract-year-2014-medication-therapymanagement-mtm-program-submission-v040513.pdf. Accessed November 29, 2016

5. Shapiro JR. CMS Memorandum. CY 2015 medication therapy management program guidance and submission instructions. May 7, 2014. Available at: https://www.cms.gov/Medicare/Prescription-Drug-Coverage/ PrescriptionDrugCovContra/Downloads/MemoContractYear2015 MedicationTherapyManagementProgramSubmission050714.pdf. Accessed November 29, 2016

6. Larrick AK. CMS Memorandum. CY 2016 medication therapy management program guidance and submission instructions. April 7, 2015. Available at: https://www.cms.gov/Medicare/Prescription-Drug-Coverage/ PrescriptionDrugCovContra/Downloads/Memo-Contract-Year-2016Medication-Therapy-Management-MTM-Program-Submission-v-040715.pdf. Accessed November 29, 2016.

7. Perlroth D, Olinger L, Marrufo G, et al. Medication therapy management in chronically ill populations: final report. Prepared for Centers for Medicare \& Medicaid Services. Contract \#HHSM-500-2011-000121/TOT0001. August 2013. Available at: http://innovation.cms.gov/Files/reports/MTM_ Final_Report.pdf. Accessed November 29, 2016.

8. Centers for Medicare \& Medicaid Services. 2016 Medicare Part D medication therapy management (MTM) programs fact sheet: summary of 2016 MTM pograms. May 4, 2016. Available at: https://www.cms.gov/Medicare/ Prescription-Drug-Coverage/PrescriptionDrugCovContra/Downloads/ CY2016-MTM-Fact-Sheet.pdf. Accessed November 29, 2016.

9. OutcomesMTM. 2015 MTM trends report. Available at: http://outcomesmtm.com/documents/2015MTMTrendsReport.pdf. Accessed November 29, 2016

10. Centers for Medicare \& Medicaid Services. CMS Medicare 2016 Part C \& D star rating technical notes. Updated September 30, 2015. pp. 67-68. Available at: http://docplayer.net/13285877-Medicare-2016-part-c-d-starrating-technical-notes.html. Accessed November 29, 2016.

11. Medicare Program; Contract Year 2015 Policy and Technical Changes to the Medicare Advantage and the Medicare Prescription Drug Benefit Programs. Proposed rule. 79 Fed Reg. 1917. January 10, 2014. Available at: https://www.federalregister.gov/documents/2014/01/10/2013-31497/ medicare-program-contract-year-2015-policy-and-technical-changes-to-themedicare-advantage-and-the. Accessed November 29, 2016.

12. Gruber J. Medication therapy management: a challenge for pharmacists. Consult Pharm. 2012;27(11):782-96.

13. McSpadden C. A new ballgame: comprehensive medication review. Consult Pharm. 2014;29(2):76-83. 